\title{
Luxationen und Luxationsfrakturen des Lisfranc-Gelenks
}

\author{
Martinus Richter
}

\section{Zusammenfassung}

Verletzungen des Lisfranc-Gelenks umfassen Frakturen, Luxationen und/ oder Luxationsfrakturen. Insbesondere die Verrenkungsbrüche sind folgenschwere Verletzungen, die sich erheblich auf die Funktion des gesamten Fußes auswirken. Da nach wie vor eine hohe Zahl von Kombinationsverletzungen primär nicht erkannt wird, kommt der korrekten Diagnostik als Grundlage der Behandlungsplanung entscheidende Bedeutung zu. Hierzu gehören Standardröntgenaufnahmen des Fußes in drei Ebenen (dorsoplantar, exakt seitlich und $45^{\circ}$-Schrägaufnahme); bei entsprechenden Verdachtsmomenten ist die Indikation für die Computertomografie inklusive multiplanarer Rekonstruktion notwendig. Die Grundprinzipien der adäquaten Behandlung sind die anatomiegerechte Rekonstruktion der Gelenkstrukturen und der geometrischen Beziehungen der medialen zur lateralen Fußsäule sowie die Erfassung und Therapie der ligamentären Instabilitäten. Werden diese Ziele nicht erreicht oder treten Komplikationen auf, sind oftmals Korrekturarthrodesen unvermeidlich.

\section{Lisfranc Fractures and Fracture Dislocations}

Injuries of the Lisfranc joint comprise fractures, dislocations and/or fracture dislocations. Fracture dislocations, in particular, represent prognostically severe lesions which compromise the function of the entire foot. A substantial number of injuries of the midfoot are still primarily overlooked. For this reason, a diagnostic algorithm is essential for the adequate detection of the injury components and for the planning of the therapeutic approach. Standard X-rays in three planes (dorso-plantar, lateral and oblique view) represent the diagnostic basis which may be supplemented by CT including multiplanar reconstructions. The principle strategy of treatment includes anatomic reconstruction of the joint structures, the geometric proportions of the medial and lateral foot columns and the diagnosis and treatment of ligamentous instabilities. If these goals cannot be achieved or in cases of complications further interventions, such as correction osteotomies and selected correction arthrodeses of the Lisfranc joint, are unavoidable.

\section{Einleitung}

Unter den Verletzungen der Fußregion verursachen besonders die Verletzungen des Lisfranc-Gelenks immer noch Probleme bei Diagnose und Behandlung und führen zu erheblicher Langzeitmorbidität.

Diese Verletzungen sind selten und treten überwiegend bei Verkehrsunfällen

OP-JOURNAL 2007; 23: 121 -126

(c) Georg Thieme Verlag KG Stuttgart • New York auf. Trotz erheblicher Verbesserungen der passiven Fahrzeugsicherheit haben sich Verletzungsinzidenz und -schwere bisher kaum verringert. Lisfranc-Gelenkverletzungen werden besonders bei Vorliegen von Begleitverletzungen bei der Primärdiagnostik häufig nicht richtig erkannt und deshalb nicht ausreichend versorgt. Die Komplikationsrate ist hoch und eine adäquate Behandlung schwierig. Anteil und Ausmaß von Langzeitmorbidität sind hoch. Luxationsfrakturen einzelner Mittelfußknochen führen aber schon zu höherer Langzeitmorbidität und sollten deshalb nicht unter- schätzt werden. Die Luxationsfraktur des gesamten Lisfranc-Gelenks ist als die häufigste schwere Mittelfußverletzung noch als selten zu bezeichnen und schwierig zu diagnostizieren. Die anatomische Reposition ist bei Lisfranc-Luxationsfrakturen besonders schwierig und erfordert normalerweise ein offenes Vorgehen. Bei verspäteter Versorgung ist die Wiederherstellung der korrekten Länge beider Fußsäulen und der Form des Längsgewölbes erheblich schwieriger. Verletzungen mit kombinierter Chopart- und Lisfranc-Luxationsfraktur sind häufig Teil sog. „Crush“-Verletzungen des Fußes mit ausgedehntem Weichteilschaden und sehr hoher Rate von Infektionen oder anderen Komplikationen. Diese Verletzungen führen zur höchsten Langzeitmorbidität aller Fußverletzungen.

\section{Hauptteil}

Topografische und funktionelle Anatomie

Das Lisfranc-Gelenk stellt die Einheit zwischen tarsalen und metatarsalen Knochen dar. Die gesamte Gelenkreihe wird durch starke Ligamente besonders plantar stabilisiert, ist aber weniger starr als bisher angenommen.

Unter der Körperlast ist eine sagittale Beweglichkeit vor allem der Randstrahlen I, IV und V um 5-17 Grad nachweisbar. Die Rigidität dieses Gelenks wird statisch durch die mechanische Stabilität der gewölbebildenden Cuneiformia bedingt. Dynamisch werden sie zusätzlich stabilisiert durch die einstrahlenden Sehnenfasern des M. tibialis posterior (medialer Steigbügel) und die quere dynamische Verspannung durch die M.peroneus-longus-Sehne, die dem lateralen Steigbügel entspricht. Die statische Verriegelung des Quergewölbes wird zudem gewährleistet durch die zahlreichen interossären Bandverbindungen. Mechanisch am stärksten verklammert ist der II. Strahl, da er durch die Verkür- 
zung des Cuneiforme II zwischen Cuneiforme I und Cuneiforme III eingeschoben ist und biomechanisch eine „Schlüsselfunktion“ hat. $\mathrm{Zu}$ beachten ist auch die Situation des I. Strahles, der a priori mehr Bewegungsfreiheit genießt, wobei einerseits 3 dynamische Stabilisatoren inserieren (M. tibialis anterior, M. tibialis posterior und $\mathrm{M}$. peroneus longus). Ein anderes besonderes Merkmal besteht darin, dass ligamentär statt eines einfachen Lig. transversale zwischen Metatarsale I/II ein Y-förmiges Band (sog. Lisfranc-Ligament) vorliegt.

\section{Pathogenese}

Pathomechanisch hat das Lisfranc-Gelenk die Schlüsselfunktion zwischen Vorfuß und Mittelfuß.

Es ist Zentrum, Schaltstelle und ein wichtiger Garant der dynamischen (I. Strahl) und statischen (II.-V.Strahl) Stabilität des Längs- und Quergewölbes des Fußes.

In Übereinstimmung mit nahezu allen Autoren luxieren die Metatarsalia durch direkte oder indirekte Gewalt am häufigsten nach dorsal, da hier der Bandapparat deutlich schwächer ist als plantar. In der 2. Luxationsebene verrenkt der I. Strahl nach medial oder mit allen 4 benachbarten Metatarsalia nach lateral. Wilson zeigte in Kadaverversuchen, dass in forcierter Vorfußpronation zunächst der I. Strahl und erst bei fortgesetzter Gewalt der II.-V.Strahl nach dorsolateral luxieren. Bei forcierter Vorfußsupination erfolgt die Luxation in umgekehrter Reihenfolge. Jeffreys zeigte experimentell, dass bei fixiertem Vorfuß und forcierter Pronation des Rückfußes die homolaterale Luxation entsteht, bei gewaltsamer Rückfußsupination die isolierte Instabilität des I. Fußstrahles.

Eigene klinische Untersuchungen und Ergebnisse der eigenen Unfallforschung haben gezeigt, dass eine Lisfranc-Gelenkluxation nach plantar eher durch direkte Gewalteinwirkung entsteht (Sturz schwerer Gegenstände auf den Vorfuß). Dorsale Verrenkungen entstehen beim Sturz auf den plantar flektierten Fuß oder durch Dezelerationstraumen bei Pkw-Insassen mit Abstützvorgängen des plantar flektierten Fußes und nachschiebender Energie.
Die Verbesserung der Fahrzeugsicherheit der 90er-Jahre führte trotz steigender Unfallschwere (Delta-v) im Vergleich zu den 80er-Jahren zu geringerer Gesamtverletzungsschwere (Injury Severity Score). Frakturen der Fußregion treten jedoch in unveränderter Häufigkeit und Verletzungsschwere (Abbreviated Injury Scale) auf.

Diese werden meist durch die Deformierung des Fußraums bei Frontalkollisionen verursacht. Besonders bei Frakturen des Mittelfußes spielt die Fußraumdeformierung die entscheidende Rolle. Eine Verringerung der Fußraumdeformierung ist daher zur Prävention essenziell. Trotz der umfangreichen technischen Untersuchung unter Einbeziehung vieler Parameter bleibt der genaue Verletzungsmechanismus besonders der Frakturen des Mittelfußes unklar. Um eine weiterführende Prävention zu ermöglichen, ist daher eine genauere Analyse des Verletzungsmechanismus im Rahmen von experimentellen Studien nötig.

Bei einer eigenen experimentellen Analyse des Verletzungsmechanismus von Frakturen des Mittelfußes konnten reproduzierbar Lisfranc-Gelenkluxationen oder -luxationsfrakturen erzeugt werden. Der analog zum realen Unfallgeschehen bei Pkw-Frontinsassen tangential zur Chopart-Gelenkfläche und senkrecht zur OSG-Fläche gerichtete Anprall verursachte völlig unerwartet höhere Kräfte im Chopart-Gelenk als im OSG, und dies besonders bei auftretenden Frakturen des Mittelfußes. Diese Unterschiede lassen sich nur mittels permanenter Druckmessung mit hoher Abtastrate erkennen. Eine Modifikation des aktuellen Fußdummys mit einer zusätzlichen Kraftmessdose im Mittelfußbereich zur Registrierung von Kräften in der Fußlängsachse ist sinnvoll. Der Dummy könnte mit dem vorgestellten Versuchsaufbau entwickelt und kalibriert werden. Mit dieser Modifikation würde eine realitätsnähere Überprüfung verbesserter Präventivmaßnahmen in Pkws ermöglicht.

\section{Diagnostik}

\section{Klinische Untersuchung}

Wichtig ist in erster Linie die klinische Diagnostik mit Prüfung der medialen und lateralen Fußsäulenlänge im Seitenvergleich, die Kontrolle auf eine spontan fixierte Vorfußabduktion oder -adduk- tion, auf Fehlstellungen in vermehrter Vorfußplantar-/-dorsalflexion, auf Störungen der aktiven Vorfußbeweglichkeit und auf lokale Druckschmerzen mit Stufenbildung. Die Prüfung der Motorik, Sensibilität und Durchblutung sowie Beurteilung des Kompartmentdrucks.

\section{Kompartmentsyndrom}

Bei etwa 25\% ist ein Kompartmentsyndrom des Fußes zu erwarten.

Bei dem Verdacht auf ein Kompartmentsyndrom sollte eine Fußkompartmentdruckmessung durchgeführt werden (z.B. mit einem Intracompartmental Permanent Pressure Monitoring System, Stryker $^{\mathrm{TM}}$ Corporation, Santa Clara, CA, USA). Bei einer Druckdifferenz von weniger als $30 \mathrm{mmHg}$ zwischen dem diastolischen Blutdruck und dem Druck im Kompartment sollte eine Faszienspaltung erfolgen.

\section{Radiologische Diagnostik}

Erst danach erfolgt die radiologische Diagnostik des Fußes mit 3 Standardaufnahmen:

1. dorso-plantare Aufnahme des ganzen Fußes mit $20^{\circ}$ kaudo-kranial gekippter Röntgenröhre,

2. exakt seitliche Projektion des gesamten Fußes,

3. $45^{\circ}$ Schrägprojektion des Mittelfußes mit den angrenzenden Gelenken (Chopart- und Lisfranc-Gelenk).

$\mathrm{Zu}$ beachten ist auf der seitlichen Aufnahme besonders die sog. Cyma-Linie als Gelenkverlauf des Chopart-Gelenks, die normalerweise exakt S-förmig geschwungen sein muss. In Zweifelsfällen sind Vergleichsaufnahmen der gesunden Seite hilfreich. Bei Verdacht auf Instabilität empfehlen sich gehaltene Aufnahmen dorso-plantar mit Rückfußvarus-/-valgusstress. Bei Impressionsfrakturen oder bei Verdacht auf Cuboidluxation sind Tomografien des Mittelfußes in 2 Ebenen sehr vorteilhaft, ggf. eine axiale $\mathrm{CT}$ und dreidimensionale $\mathrm{Re}$ konstruktion.

Weiterhin sollten Begleitfrakturen ausgeschlossen werden (in 75\% Begleitfrakturen der unteren Extremität, in $25 \%$ kombinierte Chopart-Lisfranc-Luxationsfrakturen). 


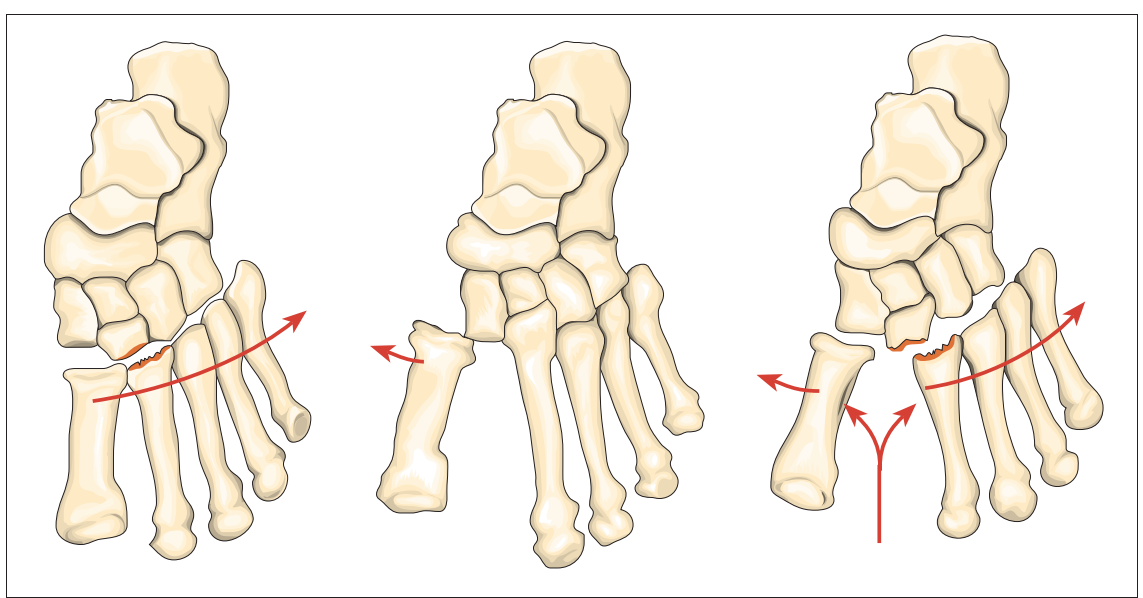

Abb. 1 Klassifikation nach Quenu und Küss [4]: homolaterale (links), isolierte (Mitte) und divergierende Luxation (rechts).

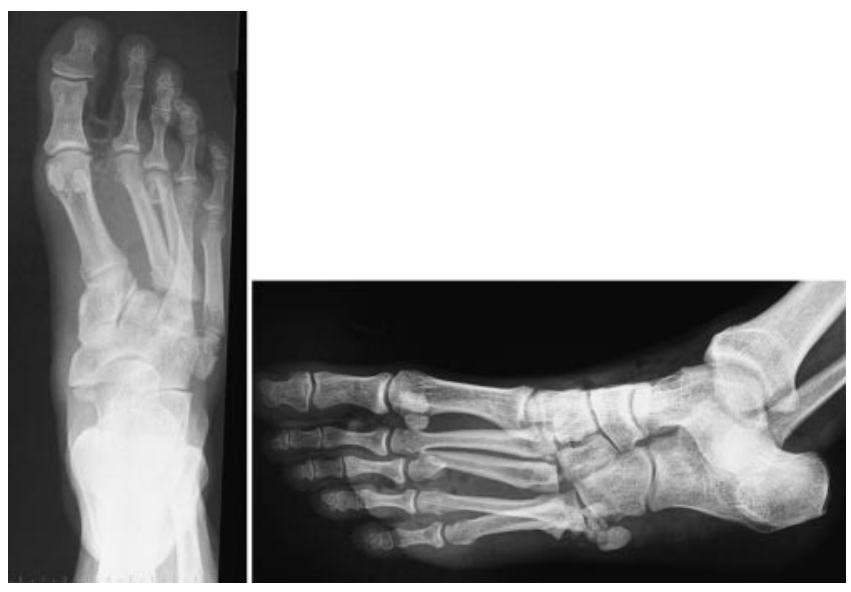

Abb. 2 Präoperative Aufnahmen: homolaterale, laterale Lisfranc-Luxationsfraktur des I. V. Strahls. Metatarsale-V-Halsfraktur.

\section{Klassifikation}

Nach Quenu und Küss werden isolierte, homolaterale und divergierende Luxationen unterschieden (Abb.1 und 2). Diese Klassifikationen wurden später von Hardcastle und Myerson modifiziert.

\section{Behandlung}

\section{Nicht operative Behandlung}

Subluxationen und komplette Luxationen im Lisfranc-Gelenk, die reponibel und danach im Gips stabil retinierbar sind, stellen klassische Indikationen zum nicht operativen Vorgehen dar.

\section{„Semioperative“ Behandlung}

Alle Luxationsformen, die zwar geschlossen anatomisch reponibel, aber im Gips nicht stabil retinierbar sind, sollten mit perkutanen Spickdrähten temporär fixiert und zusätzlich im Gips-
- Regionalanästhesie (spinal/epidural/ popliteal) oder „Vollnarkose“

- Antibiotikaprophylaxe je nach häuslichem Standard (z.B. Cephalosporine 3. Generation).
Tisch und OP-Set

- 3,5-mm-Kortikalis- und Spongiosaschrauben mit Stahl oder Titaniumlegierung

- 1,0-2,5-mm-Spickdrähte

- Standardosteosyntheseset, je nach internem Standard

- Röntgentisch

- Röntgengerät

- Karbontisch und dreidimensionales Röntgengerät, wenn verfügbar (z.B. ARCADIS, Siemens AG, Erlangen).

\section{Tisch Setting}

- Instrumente stehen am Fußende des Tisches

- Röntgengerät sollte auf der kontralateralen Seite zur Verfügung stehen.

Patientenlagerung

- Rückenlage

- Blutsperre wird nicht empfohlen, da hohes Risiko einer ischämischen Wundnekrose.

\section{Abdeckung}

- Reinigung/steriles Abwaschen des Unterschenkels und des ganzen Fußes mit Desinfektionslösung, wobei eine gründliche Reinigung interdigital erfolgen sollte

- Steriles Abdecken ab der Mitte des Unterschenkels, zwischen Knie und Sprunggelenk

- Tape um jeden einzelnen Zeh (Erleichtert die Reposition).

Operativer Zugang

- Es erfolgt in der Regel eine lange mediane Inzision, die zwischen dem II. und III.Strahl in Höhe des Sprunggelenkes beginnen und in Höhe des Metatarsophangealgelenkes enden sollte (Abb. 3)

- Schonung des Gefäß- und Nervenbündels zwischen dem I. und II. Strahl (A. dorsalis pedis, N. peroneus profundus)

- Freilegung und Mobilisation der Sehnen des M. hallucis longus und M. extensor digitorum. Die Mobilisation ermöglicht eine Verschiebung der Sehnen nach lateral oder medial.

- Schonung der Extensorenloge

- Unter den Sehnen der Extensoren ist von dorsal das Lisfranc-Gelenk aufzufinden

- Schonung der dorsalen Gelenkkapsel 


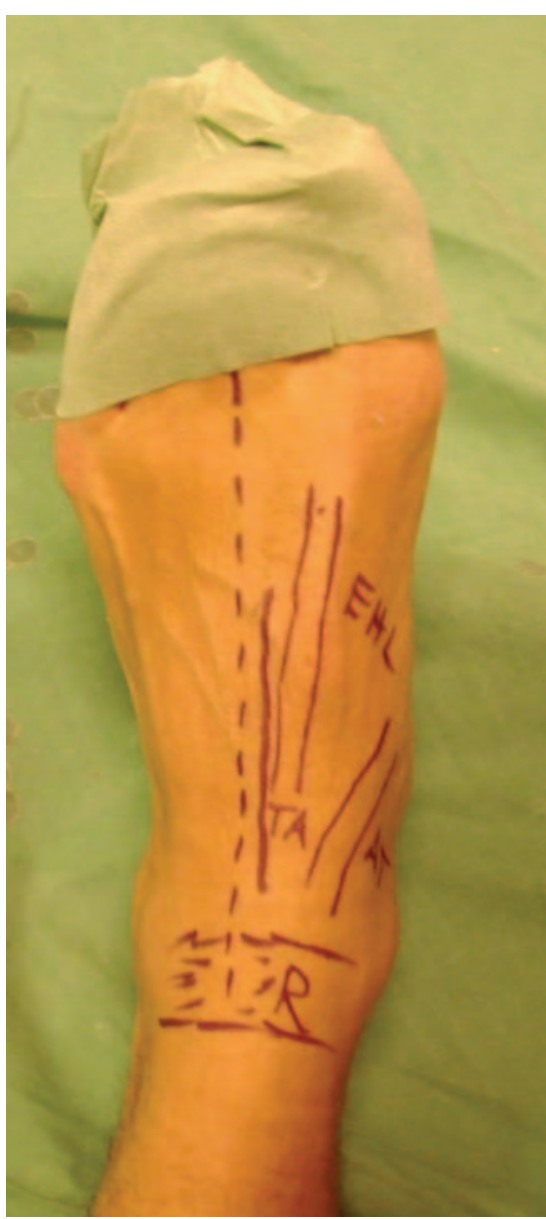

Abb. 3 Dorsomedialer Zugang zum LisfrancGelenk. Gestrichelte Linie: Zugang; R: Retinaculum; EHL: Sehne des M. extensor hallucis; TA: A. dorsalis pedis; AT: Sehne des M. tibialis anterior.

Reposition und interne Fixation

Die offene Reposition beginnt am Metatarsale II.

- Da die Lage des Metatarsale II die Funktion eines Schlusssteines zwischen dem lateralen Cuneiforme und dem medialen Cuneiforme hat, sollte zwischen der Basis des Metatarsale II und dem medialen Cuneiforme eine exakte anatomische Reposition der Gelenkflächen erfolgen. Eine Röntgenkontrolle wird empfohlen. Das Metatarsale II sollte zunächst mit kleinen Spickdrähten anatomisch aufgebaut werden. Danach erfolgt die Reposition zum Cuneiforme II und die temporäre Transfixation mit zwei seitlich eingebrachten Spickdrähten von der Basis bis in das Cuneiforme II. Erst jetzt kann die stabile Transfixation mit der 3,5-mm-Kortikalisschraube erfolgen, wobei mit dem
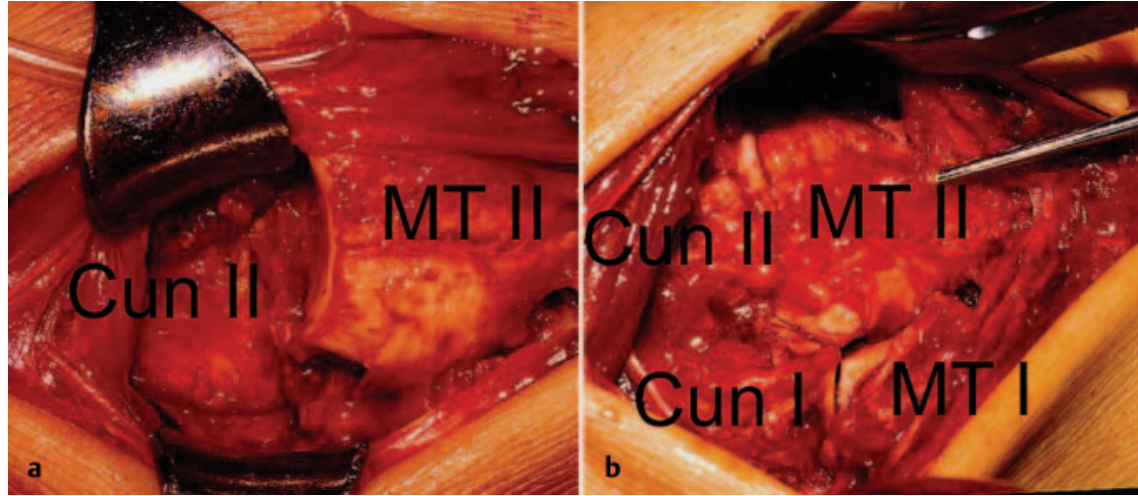

Abb. 4a und b Homolaterale, laterale Lisfranc-Luxationsfraktur des I. - V. Strahls. MetatarsaleV-Halsfraktur. Geschlossene Reposition und interne K-Draht-Fixation mit temporärer Transfixation von Metatarsale V zu Metatarsale III und IV. a Intraoperative Aufnahme der dislozierten Metatarsale-II-Basis vor der Reposition. b Intraoperative Aufnahme nach Reposition des Metatarsale II und internen Fixation mit einem 2,0-mm-K-Draht.

Luer zunächst eine kleine Grube am dorsalen Metatarsale $1,5 \mathrm{~cm}$ distal des Gelenkes geschaffen wird, damit der Schraubenkopf hier gut eintauchen kann. Die Bohrung selbst erfolgt ganz tangential zum Metatarsale mit einem 2,5-mm-Bohrer.

- Danach sollte die Reposition am Metatarsale I erfolgen. Zur internen Stabilisierung zwischen Metatarsale I und dem medialen Cuneiforme sollten ein 2-mm-K-Draht oder eine 3,5-mm-Zugschraube verwendet werden.

- Die offene Reposition und interne Fixation der Metatarsale III - V erfordert ein schräges Einbringen von Spickdrähten. Manchmal besteht die Notwendigkeit einer zusätzlichen Transfixation zwischen einzelnen Metatarsalen, um eine ausreichende Stabilität zu erreichen (Abb. $\mathbf{4}$ bis $\mathbf{7}$ ).

- Bei erheblicher Gelenkzerstörung sollte eine primäre Arthrodese in Betracht gezogen werden, da damit bessere Langzeitergebnisse als mit einer sekundären Arthrodese erzielt werden können. Dabei muss nicht eine komplette Gelenkversteifung erfolgen. Die funktionellen Ergebnisse nach isolierter Versteifung der medialen oder lateralen Säule sind besser als nach kompletter Versteifung. Das operative Vorgehen ist analog zur temporären Schraubentransfixation. Abweichend hiervon muss eine komplette Entknorpelung der zu versteifenden Gelenke erfolgen und die Schrauben sollten als Zugschrauben eingebracht werden. Zur Stabilitätserhöhung kann eine Verschraubung der Cuneiformia in mediolateraler Richtung hilfreich sein.
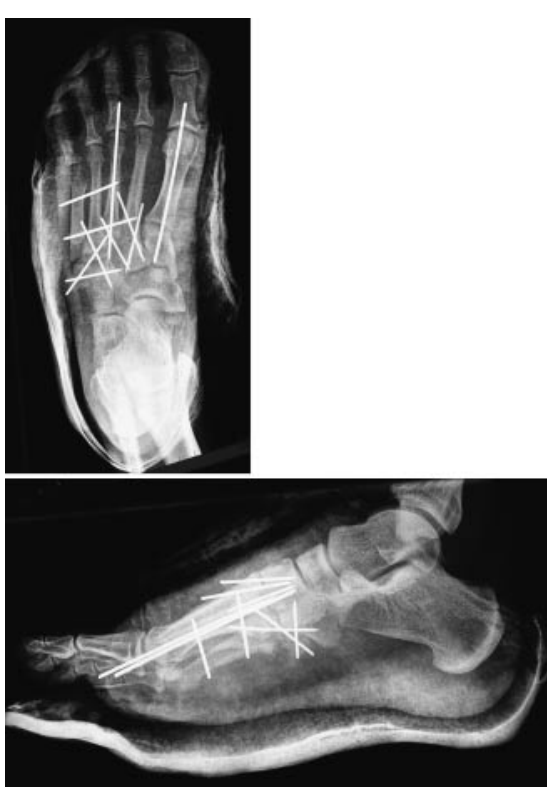

Abb. 5 Postoperative Aufnahmen. Homolaterale, laterale Lisfranc-Luxationsfraktur des I. - V. Strahls. Metatarsale-V-Halsfraktur. Geschlossene Reposition und interne K-Draht-Fixation mit temporärer Transfixation von Metatarsale V zu Metatarsale III und IV.

\section{Wundverschluss}

- Gründliche Spülung und Blutstillung

- Einlage einer Redon-Drainage (12 F) und Adaptation der subkutanen Faszie (2.0 PDS/Vicryl)

- Verschluss der Haut mit monofilem, nicht resorbierbarem Nahtmaterial

- Ist ein primärer Hautverschluss nicht möglich, so sollte der Defekt temporär mit Epigard gedeckt werden. Innerhalb von 1 oder 2 Wochen muss dann ein sekundärer Hautverschluss möglich sein, und eine Deckung mit Spalthaut ist nicht notwendig. 
- Anlage eines Unterschenkelspaltgipses.

\section{Postoperative Versorgung}

- Entfernung der Redon-Drainage nach 48 Stunden.

- Nach Abschwellung: Anlage eines geschlossenen Unterschenkelgipses

- Frühfunktionelle Teilbelastung mit $15 \mathrm{~kg}$ und Physiotherapie.

\section{Kontrolluntersuchungen}

- Wiedervorstellung nach 6 und 12 Wochen mit Röntgenkontrolle

- Nach Röntgenkontrolle nach 6 Wochen: Entfernung des Gipses und temporärer Implantate und Übergang zur Vollbelastung

- Nach klinischer und radiologischer Dokumentation eines guten Heilungsprozesses muss eine weitere Nachuntersuchung nur auf Bitte um Wiedervorstellung durch einen niedergelassenen Chirurgen erfolgen

- Wiedervorstellung mit Röntgenbildern nach 1 Jahr zum Ausschluss einer posttraumatischen Arthrose.

\section{Prognose}

Rein ligamentäre Verletzungen des Mittelfußes verheilen unter nicht operativer Behandlung in der Regel folgenlos, knöcherne Bandausrisse des Lig. bifurcatum, vor allem mit Gelenkbeteiligung, neigen unter nicht operativer Behandlung zur schmerzhaften pseudarthrotischen Fehlverheilung.
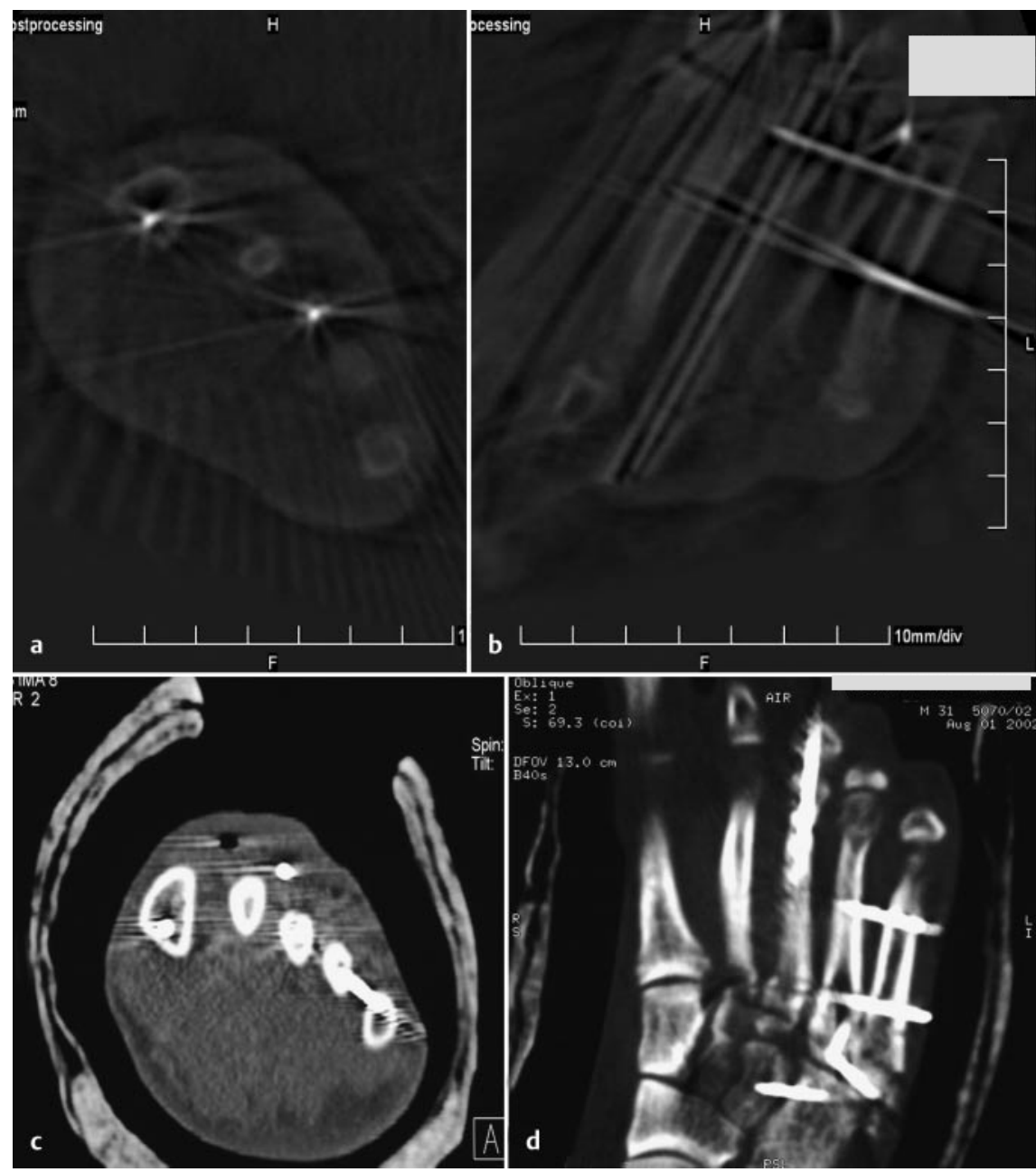

Abb. 6a bis d Homolaterale, laterale Lisfranc-Luxationsfraktur des I. - V. Strahls. MetatarsaleV-Halsfraktur. Geschlossene Reposition und interne K-Draht-Fixation mit temporärer Transfixation von Metatarsale V zu Metatarsale III und IV. a, b Intraoperative Aufnahme mit einem Röntgengerät für dreidimensionale Aufnahmen (ISO-C-3D bzw. ARCADIS, Siemens AG, Erlangen). c, d Postoperatives CT.
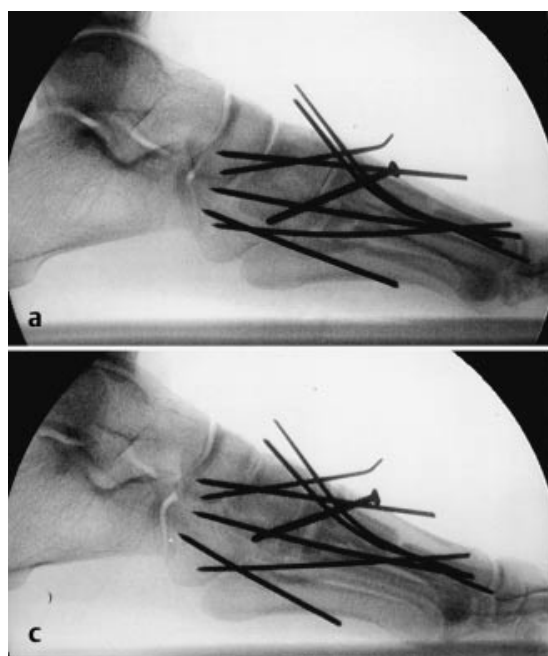
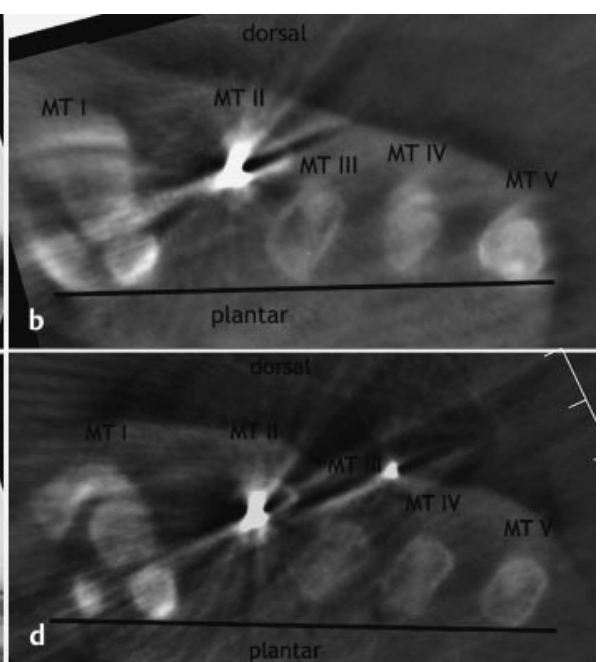

Abb. 7a bis d Fall mit sinnvoller intraoperativer dreidimensionaler Bildgebung (anderer Fall als Abb. $\mathbf{2}$ bis $\mathbf{6})$. a Röntgenbildverstärkerbild nach offener Reposition und interner Fixation einer Lisfranc-Luxationsfraktur. Auf diesem Bild erkannte der Operateur keine Fehlstellung. Dann Scan mit ARCADIS und axiale Reformation durch die Metatarsaleköpfchen (b). Hier zeigt sich ein deutliches Tiefertreten des Metatarsale-III-Köpfchens, was für korrekturpflichtig gehalten wurde. Es erfolgte dann im selben Eingriff eine erneute Reposition und interne Fixation des Metatarsale III im Lisfranc-Gelenk. Auf dem seitlichen Röntgenbildverstärkerbild danach (c) ist kein relevanter Unterschied zu vorher (a) zu erkennen. Die erneute dreidimensionale Darstellung (d) zeigt aber eine nun wesentlich bessere Stellung des Metatarsale-III-Köpfchens. 
Alle relevanten Gelenkverwerfungen und/oder Verkürzungen der medialen bzw. lateralen Fußsäule führen ohne primär-rekonstruktiven Eingriff in der Regel zur posttraumatischen Arthrose, Valgus-/Varusfehlstellung des Vorfußes, Hemmung der Pronation bzw. Supination und/oder der Dorsal-/Plantarflexion.

Nach Fraktur des Naviculare besteht auch bei primärer korrekter Versorgung die Gefahr der aseptischen Nekrose. Bei primärer Knorpelzerstörung sind posttraumatische Arthrosen relativ häufig zu sehen, die in der Regel aber besser toleriert werden als in anderen Gelenkabschnitten des Fußes. Die Prognose hängt auch besonders vom Ausmaß des initialen Weichteilschadens ab. Rasche Kompartmentspaltung, anatomische Rekonstruktion und temporäre stabile Transfixation sind bei hohem Weichteilschaden günstig. Verzögerte Kompartmentspaltung, unzureichende konservative oder semioperative Behandlung mit perkutaner Spickdrahtfixation können Steifigkeit, Kontrakturen und Fehlstellungen verursachen, die dann oftmals nur durch korrigierende Eingriffe beherrschbar sind.

\section{Schlussfolgerung}

Diagnostik und Behandlung von Frakturen, Luxationen oder Luxationsfrakturen des Lisfranc-Gelenks sind auch heute noch problematisch. Besonders Luxationsfrakturen beeinträchtigen die gesamte Fußfunktion und führen $\mathrm{zu}$ erheblichen Langzeitfolgen, welche sich am ehesten durch frühe anatomische (offene) Reposition und suffiziente (interne) Retention minimieren lassen. Angesichts des typischen klinischen Verlaufs mit schweren Langzeitfolgen, auch bei optimaler Behandlung, spielt die Verletzungsprävention von Lisfranc-Verletzungen eine vordringliche Rolle. Aufgrund der überwiegenden Entstehung dieser Verletzungen bei Verkehrsunfällen und insbesondere bei Pkw-Insassen sollten hier präventive Maßnahmen überprüft und ggf. modifiziert werden.

\section{Literatur}

${ }^{1}$ Hardcastle PH, Reschauer R, Kutscha-Lissberg E, Schoffmann W. Injuries to the tarsometatarsal joint. Incidence, classification and treatment. J Bone Joint Surg [Br] 1982; 64: 349-356

2 Lynch JRS, Cooperstein LA, Di Gioia AM. Plantar medial subluxation of the medial cuneiform: case report of an uncommon variant of the Lisfranc injury. Foot Ankle Int 1995; 16: 299-301

${ }^{3}$ Myerson MS, Fisher RT, Burgess AR, Kenzora JE. Fracture dislocations of the tarsometatarsal joints: end results correlated with pathology and treatment. Foot Ankle 1986; 6: 225 242

${ }^{4}$ Quenu E, Küss G. Etudes sur les luxations du metatarse. Rev Chir 1909; 39: 1093 - 1134

5 Richter M, Thermann H, Hufner T, Krettek C. Aetiology, Treatment and Outcome in Lisfranc Joint Dislocations and Fracture Dislocations. Foot Ankle Surg 2002; 8: 21 - 32
${ }^{6}$ Richter M, Thermann H, Wippermann B, Otte D, Schratt HE, Tscherne H. Foot fractures in restrained front seat car occupants: a longterm study over twenty-three years. J Orthop Trauma 2001; 15: 287-293

7 Richter M, Wippermann B, Krettek C, Schratt E, Hufner T, Thermann H. Fractures and fracture dislocations of the midfoot - occurence, causes and long-term results. Foot Ankle Int 2001; 22: $392-398$

8 Richter M, Wippermann B, Thermann $\mathrm{H}$, Schroeder G, Otte D, Troeger HD, Krettek C. Plantar impact causing midfoot fractures result in higher forces in Chopart's joint than in the ankle joint. J Orthop Res 2002; 20: 222 232

${ }^{9}$ Zwipp H. Chirurgie des Fußes. Wien, New York, 1994

10 Zwipp H, Dahlen C, Randt T, Gavlik JM. Komplextrauma des Fußes. Orthopäde 1997; 26: 1046-1056

\section{Prof. Dr. med. Martinus Richter} Chefarzt

II. Chirurgische Klinik (Unfallchirurgie, Orthopädie und Fußchirurgie) Klinikum Coburg

Ketschendorfer Straße 33 96450 Coburg 